\title{
Psychological impact of medical and surgical interventions for Gender Dysphoria - A Literature Review
}

\section{Background and aims}

The definition of Gender Dysphoria (GD) encompasses a marked difference between the individual's expressed/experienced gender and the gender others would assign him or her (DSM-5). Among others, issues related to body-image correlate with a lack of confidence and self-esteem that compromise the subject's well-being and dayto-day life. According to current data, these patients present a higher rate of anxiety, depression, suicide and self-harm when compared to the general population (GP).

\section{Materials and Methods}

A literature review was performed, based on a Pubmed $^{\circledR}$ search for articles published in the last 5 year, with topics: "gender dysphoria", "hormonal treatment", "surgical treatment".

Results

\begin{tabular}{|c|c|c|c|c|c|c|c|}
\hline $\begin{array}{l}\text { Authors (year) } \\
\text { Country }\end{array}$ & $\begin{array}{l}\text { Number of } \\
\text { trans participants/ } \\
\text { dlagnosis/ } \\
\text { mean age at } \\
\text { assessment }\end{array}$ & $\begin{array}{l}\text { Treatment status: } \\
\text { (on CHT or } \\
\text { post-GCGS) }\end{array}$ & Study design & $\begin{array}{l}\text { Comparative } \\
\text { groups }\end{array}$ & $\begin{array}{l}\text { Outcome } \\
\text { measure }\end{array}$ & Prevalence in trans & Conciusion \\
\hline $\begin{array}{l}\text { Fisher et al. }(2013)^{b} \\
\text { Italy }\end{array}$ & $\begin{array}{l}32.3 \text { years FtM } \\
47.9 \text { years MtF } \\
92 \mathrm{MtF} \\
48 \mathrm{FtM} \\
\text { DSM-IV-TR } \\
32.6 \text { years }\end{array}$ & $\begin{array}{l}\text { CHT } 69.8 \% \text { GCGS } \\
22.1 \%\end{array}$ & $\begin{array}{l}\text { Single centre } \\
\text { (Gender clinic) } \\
\text { Cross-sectlonal }\end{array}$ & No & $\begin{array}{l}\text { SCID-1-HI } \\
\text { SCl-90R }\end{array}$ & $\begin{array}{l}\text { Axis I disorders } 18.7 \% \\
\text { Mood and adjustment disorder } 10.8 \% \\
\text { Anxlety disorder } 5 \% \\
\text { Axis II dlsorders } 4.3 \%\end{array}$ & Gender: no difference \\
\hline $\begin{array}{l}\text { Gorin-Lazard et al. } \\
(2013)^{\mathrm{c}} \\
\text { France }\end{array}$ & $\begin{array}{l}31 F T M \\
36 \text { MtF } \\
\text { DSM-IV-TR } \\
35.1 \text { years }\end{array}$ & $\begin{array}{l}\text { CHT } 73.1 \% \\
\text { GCGS NR }\end{array}$ & $\begin{array}{l}\text { Muiti-centre } \\
\text { (Gender cllinics) } \\
\text { Cross-sectional }\end{array}$ & $\begin{array}{l}\text { Trans with and } \\
\text { without } \mathrm{CHT}\end{array}$ & $\begin{array}{l}\text { BDI } \\
\text { SSEI }\end{array}$ & $\begin{array}{l}\text { Depression and self-esteem: } \\
\text { Trans on CHT less depressive symptoms, } \\
\text { better self esteem }\end{array}$ & $\begin{array}{l}\text { NA } \\
\text { Umitation: } \\
\text { Pre-/post groups were not the same }\end{array}$ \\
\hline $\begin{array}{l}\text { Davey et al. (2014) } \\
\text { UK }\end{array}$ & $\begin{array}{l}63 \mathrm{MtF} \\
40 \mathrm{FtM} \\
\mathrm{KCD}-10 \\
45.7 \text { years }\end{array}$ & $\begin{array}{l}\text { CHT } 78.6 \% \\
\text { GCGS } 16.5 \%\end{array}$ & $\begin{array}{l}\text { Single centre } \\
\text { (Gender cllic) } \\
\text { Cross-sectional }\end{array}$ & $\begin{array}{l}\text { CC } 103 \\
\text { Controlled by age }\end{array}$ & SCL-9OR & $\begin{array}{l}\text { Psychopathology: } \\
\text { SCL-90R scores higher in trans }\end{array}$ & $\begin{array}{l}\text { Social support did not significantiy predict } \\
\text { psychopathology }\end{array}$ \\
\hline $\begin{array}{l}\text { Duisin et al. (2014) } \\
\text { Serbla }\end{array}$ & $\begin{array}{l}21 \text { MtF } \\
9 \text { FtM } \\
\text { DSM-IV-TR } \\
30.4 \text { years }\end{array}$ & $\begin{array}{l}\text { CHT NR } \\
\text { GCGS O\% }\end{array}$ & $\begin{array}{l}\text { Single centre } \\
\text { (Gender clinic) } \\
\text { Cross-sectiona! }\end{array}$ & CC 30 & $5 \mathrm{CIO}-11$ & $\begin{array}{l}\text { Axis-1l diagnosis } 66.6 \% \text { (most } \\
\text { frequent paranold and avoidant) }\end{array}$ & $\begin{array}{l}\text { Difference: GIO group more Axis-ll } \\
\text { disorders compared to CC group } \\
\text { Gender: MtF more psychopathology } \\
\text { compared to FtM }\end{array}$ \\
\hline $\begin{array}{l}\text { Fisher et al. (2014) } \\
\text { Italy }\end{array}$ & $\begin{array}{l}59 \text { FtM } \\
66 \text { MtF } \\
\text { DSM-IV-TR } \\
28.7 \text { years FtM } \\
33.1 \text { years MtF }\end{array}$ & $\begin{array}{l}\text { CHT O\% } \\
\text { GCGS NR }\end{array}$ & $\begin{array}{l}\text { Mult-centre } \\
\text { (Gender clinics) } \\
\text { Cross-sectlonal }\end{array}$ & $\begin{array}{l}\text { Trans with } \\
\text { and without } \mathrm{CHT}\end{array}$ & $\begin{array}{l}\text { SCL-90R } \\
\text { BUT GSI }\end{array}$ & $\begin{array}{l}\text { Psychopathology: } \\
\text { No difference between both on SCL-9oR } \\
\text { BUT GSI: MisF with CHT group had less } \\
\text { body uneasiness than not treated group }\end{array}$ & $\begin{array}{l}\text { Body uneasiness effectively diminished } \\
\text { with CHT } \\
\text { Limitation: Pre-/post groups were } \\
\text { not the same }\end{array}$ \\
\hline $\begin{array}{l}\text { Heylens et al, } \\
\text { (2014a) } \\
\text { Belglum } \\
\text { Germany } \\
\text { Netherlands } \\
\text { Norway }\end{array}$ & $\begin{array}{l}182 \text { MefF } \\
123 \mathrm{FtM} \\
\text { DSM-IV-TR } \\
22.8-31.2 \text { years } \\
\text { FtM } \\
21.6-36.5 \text { years } \\
\text { MtF } \\
\text { (Depends } \\
\text { country) }\end{array}$ & $\begin{array}{l}\text { CHT O\% } \\
\text { GCGS O\% }\end{array}$ & $\begin{array}{l}\text { Multicentre } \\
4 \text { countries Cross. } \\
\text { sectional }\end{array}$ & No & $\begin{array}{l}\text { MINI } \\
\text { SCIO-\|I }\end{array}$ & $\begin{array}{l}\text { Ax's I diagnosis (current) } 38 \% \text { Affective } \\
\text { probiems } 27 \% \\
\text { Anxiety problems } 17 \% \\
\text { Axis I (eurrent and iffetime) } 70 \% \\
\text { Affective problems } 60 \% \\
\text { Anxiety problems } 28 \% \\
\text { Ax's II dlagnosis } 15 \%\end{array}$ & Gender, age of onser No differences \\
\hline $\begin{array}{l}\text { Mazaherl Meybodl } \\
\text { et al. (2014a) } \\
\text { Iran }\end{array}$ & $\begin{array}{l}47 \mathrm{MtF} \\
36 \mathrm{FtM} \\
\text { DSM-IV-TR } \\
\text { Age: NA }\end{array}$ & $\begin{array}{l}\text { CHT 92.9\% } \\
\text { GCGS } 09 \%\end{array}$ & $\begin{array}{l}\text { Single centre } \\
\text { (Gender ellinic) } \\
\text { Cross-sectional }\end{array}$ & No & $5 \mathrm{ClO}-1$ & $\begin{array}{l}\text { Axis-1 diagnosis } 62.7 \% \\
\text { Major depressive disorder (33.7\%6) } \\
\text { Specific phobla (20.5\%) } \\
\text { Adjustment dlsorder (15.7\%) }\end{array}$ & $\begin{array}{l}\text { High prevaience of Axís I diagnosis } \\
\text { Uimitation: } \\
\text { No controls }\end{array}$ \\
\hline $\begin{array}{l}\text { Mazaher| Meybodl } \\
\text { et al. (2014b) } \\
\text { Iran }\end{array}$ & $\begin{array}{l}39 \mathrm{M}: F \\
31 \mathrm{FtM} \\
\text { DSM-IV-TR } \\
\text { Age: NA }\end{array}$ & $\begin{array}{l}\text { CHT 92.9\% } \\
\text { GCGS } 096\end{array}$ & $\begin{array}{l}\text { Single centre } \\
\text { (Gender clinic) } \\
\text { Cross-sectional }\end{array}$ & No & MCMI-II. & Axis I| diagnosis $81.4 \%$ (57.1\% nareissistic) & $\begin{array}{l}\text { High prevalence of Axís II diagnosis } \\
\text { Umitation: } \\
\text { No controls }\end{array}$ \\
\hline
\end{tabular}

BDI, Beck depression Inventory; BUT-GSI, Body Uneasiness Test Global Severity Index (the total score of BUT); CC, CIs controls; CHT, =cross-sex hormonal treatment; DES, Dissoclative Experience Scale; DDIS, Dissociative Disorders Interview Schedule; FtM, female-to-male subjects, trans men; GAF, Global Assessment of Functioning Scaie; GCGS, gender confirmation genital surgery; GD, Gender dysphoria; GID, gender identity disorder;

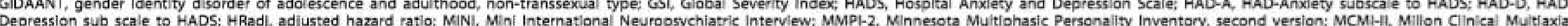
Inventory, second verslon; MtF, male to female subjects, trans women: NR, not reported; RSE, Rosenberg Self-Esteem scale; SADS, Soclal Avoidance and Distress Scale; SCID-1 and II, Structured CIInical Interview for DSM-1V, Axls I and II disorders; SCID-D, Structured Clinical Intervew for DSM-IV-Dissociative Disorders; SCL-90R, Symptom Checklist-90 (revised); SES, Self-Esteem SCale; SF-36, Short Form 36-item Questionnaire; SSEl, Soclal Self -Esteem Inventory.

a.rstudies using the same data.

\section{Conclusions}

A multidisciplinary therapeutic approach to GD is of the upmost importance, as it should involve medical, surgical and psychological counseling. Even though the first two may be crucial to obtain an appearance that is compatible with the individual's identity, the latter is essential during the various stages of intervention, because it leads to a better understanding of the subject's GD, while allowing the prevention or early diagnosis of psychiatric disorders. 